

\title{
Bringing tacit knowledge back to contributory and interactional expertise: A reply to Goddiksen
}

Luis Reyes-Galindo ${ }^{\mathrm{a}}$, Tiago Ribeiro Duarte ${ }^{\mathrm{b}}$

\begin{abstract}
We analyse a recent paper by Goddiksen (2014) where the author raises questions about the relationship between authorship, attribution and Collins \& Evans' concept of contributory and interactional expertise. We then highlight recent empirical work in the sociology of climate change science that has made similar points in order to clarify how authorship, division of labour and contribution are handled in real scientific settings. Despite this, Goddiksen's critique of both contributory and interactional expertise is ultimately ineffective because it rests on a misguided attempt to de-socialise these concepts. We conclude by stressing the importance of collective tacit knowledge acquisition through immersion as a critical step in becoming a full-blown contributory or interactional expert.
\end{abstract}

Keywords: interactional expertise, contributory expertise, tacit knowledge, enculturation, immersion.

\section{Introduction: some definitions}

In a recent paper Goddiksen (2014) proposes that contributory and interactional expertise are not clearly defined and require clarification. This is a useful endeavour as Collins, Evans and collaborators have been working on these concepts for more than a decade and, as with any developing concept, multiple definitions of both types of expertise have been put forward in different contexts. In the original 'Third Wave' paper (Collins \& Evans, 2002: 254) interactional expertise is defined as "enough expertise to interact interestingly with participants of a domain and carry out a sociological analysis". Improving on that, Collins \& Evans (2007: 28) define interactional expertise as "expertise in the language of a specialism in the absence of expertise in its practice". A recent paper (Collins 2011) pointed out that all contributory experts have interactional expertise in their domain of practices, with a new category created for individuals who only have interactional expertise in a domain of practice but no contributory expertise: special interactional experts (e.g. sociologists of science who spend a long time immersed in a scientific domain and become accomplished speakers of its language).

Goddiksen does not use the most recent 2011 elaboration to carry out his critique. This is unfortunate because the Studies in Expertise and Experience (SEE) programme now has a well developed and empirically supported conceptual mapping of expertise-related concepts: expertise is the mastery of the tacit knowledge of a domain of practice, with interactional expertise being mastery of the domain's language and contributory expertise being the ability to competently engage in the practices of that domain. From the very start, however, Goddiksen's definition of

\footnotetext{
a Cardiff University, School of Social Sciences, Glamorgan Building, King Edward VII Avenue, Cardiff, CF10 3WT, Wales, United Kingdom. Email: LuisReyes@,ciencias.unam.mx

b University of Brasília, Campus Universitário Darcy Ribeiro/UnB, Instituto de Ciências Sociais Departamento de Sociologia, Prédio Multiuso II, Brasília/DF, Brazil , 70910-900. Email: 
expertise is at odds with Collins and Evans' 2011 definition: "I take an expertise to be a set of skills that enable a person to perform certain tasks that are of importance to a wider community in a way that benefits this community" (2014:2). But, as pointed out above, Collins and Evans have defined expertise as mastery of the tacit knowledge shared by the members of a domain of practice. Whether the possession of expertise will lead to something beneficial to a domain or not is a whole different matter. Indeed, opposing members of core-sets tend to be quite vocal about how much harm their opponents are causing to the field in spite of their high levels of expertise (Collins 1992, 1998).

\section{Goddiksen on contributory expertise}

Goddiksen's first step is to try to clarify the concept of contributory expertise by attempting to work out what type of contribution would make someone a contributory expert. For example, he asks whether a special interactional expert who engages in a conversation with the contributory experts of the relevant domain and makes useful suggestions that help solve a problem should be regarded as a contributory expert. He also points out that although a practicing chemist or a physics master student could be useful in a physics lab, they would not be able to fully perform as competent researchers, so that being able to engage with some of the practices of a domain is not enough to be a contributory expert. This flexing and tracing of the boundaries of contributory expertise in ambiguous cases like students or lab technicians could lead to a useful examination of the demarcation criteria for this category.

Goddiksen first posits epistemological criteria to identify contributory experts, arguing that contributory experts should be able to make contributions that lead to the progress of the field, even while acknowledging that there is a complex philosophical debate on what scientific progress means. Indeed, given the well-known debates in both the philosophy and sociology of science of the previous century it is difficult to see what concept of progress should be used here. Furthermore, as Collins and Evans' definition of expertise does not mention contributions that benefit or lead to the progress of a domain, reviving an intractable epistemological debate does not seem to be a very tempting move.

The second criterion on offer is much more promising. Goddiksen argues that a contributory expert has to be able to make contributions to a domain that would entitle him or her to authorship of scientific papers. This point strongly resonates with work recently carried out by Duarte (2013) examining collaborations in climate change science. The research shows that the notion of contribution can be split into two categories: standardised contributions and domainlanguage based contributions. The former refers to practices that anyone who does not speak the language of a domain could make. For example, in paleoclimatology laboratories, technicians perform tasks such as washing sedimentary cores and preparing samples to be run in mass spectrometers for which no understanding of the theoretical side of paleoclimatology is needed. Domain-language-contributions refer to those that require mastery of the language of a domain. For example, to be able to interpret paleoclimatology data it is essential to have an in-depth knowledge of the principles behind the production of the data at hand, about the history of the Earth system, and about its main mechanisms of change. Interestingly, as work in progress (Duarte $2014 \mathrm{a} ; 2014 \mathrm{~b}$ ) points out, in actual scientific practice only domain-language contributions lead to authorship in paleoclimatology. It is worth noting, however, that the publication criterion could in some cases encompass some experts whose expertise was primarily interactional - such as the managers of large scientific projects.

(Collins and Sanders, 2007). Further empirical work is therefore necessary to clarify how to distinguish the special interactional experts from the contributory experts in published papers and these results would be important in further developing more solid demarcation criteria for contributory expertise within the larger SEE programme. 
Pre-print: Accepted for publication in Studies in History and Philosophy of Science Part A, November 2014.

Interactional expertise

Further on, Goddiksen makes a distinction between Collins and Evans' concept of interactional expertise and what he refers to as 'imitational expertise'. For the former, the definition is nearly the same as Collins and Evans', centred on "the ability to speak the language of the community to a relatively high degree". The latter consists of the ability to pass an Imitation Game (IG). ${ }^{1}$ Goddiksen argues that for one to pass an IG one would 'only' have to answer the questions in a way that is indistinguishable from an expert in the relevant domain, different from an interactional expert, who, in his account, could attribute different meanings to objects, concepts, etc. and still interact with members of another community.

Nevertheless, Collins and Evans would not use the qualification "relatively", as they apply the notion of interactional expert only to individuals with full command of a domain language. This is because the concept of interactional experts, however, was created to encompass individuals who share the same language and consequently the same frame of meaning when it comes to a given domain of practice. Goddiksen instead tries to place interactional expertise alongside other STS frameworks created to think about collaboration between different expert communities, such as interlanguages (Galison 1997) or boundary objects (Star \& Griesemer, 1989) that do not rely on shared meanings (Collins, Evans \& Gorman 2007; Ribeiro 2007). Goddiksen assumes that Collins and Evans argue that effective collaboration can only happen in contexts where interactional expertise is the mechanism to bridge the gaps between different communities. This is certainly not the case, as Collins, Evans \& Gorman (2007) have clearly pointed out. While there may still be room to debate how interactional and imitational expertise overlap, the discussion would need to be centred on the understanding that an interactional expert has, by definition, access to the frames of meaning that delimit an esoteric culture. ${ }^{2}$

The tacit dimension(s)

So far, one could argue that these are only inconsistencies in terminology between the latest definitions of SEE and Goddiksen's usage. Nevertheless, we will argue that the second half of his paper suggests that the divergences between Goddiksen's and SEE's conceptualisation of interactional expertise run much deeper and are tied to a very different philosophical understanding of this concept. Additionally, Goddiksen's proposal clashes with the empirical research that underlies the whole expertise programme.

Goddiksen gives a brief description of how one becomes a scientist: a student starts out in a bachelor's course in which the basic technical knowledge and vocabulary within a large-scale discipline (viz. physics) is taught by practicing research scientists, but he makes the point that more often than not they interact more with TA's who are more likely to be "advanced interactional experts". Further specialisation means that they come into contact with experts from other areas of research, so that "they learn parts of the language of other specialisms" and that they therefore acquire "some degree of expertise in related domains." Finally, it is claimed that "the more tacit 'tricks of the trade' are learned through theoretical and practical exercises." Through this, he claims that interactional expertise is in fact sometimes gained without substantial contact with contributory experts but this means he has shifted into a framework that thinks of expertise as coming from organised education rather than socialisation into bodies of tacit knowledge. Goddiksen correctly points out that "Collins and Evans argue that gaining interactional expertise with respect to a given domain requires immersion in the linguistic practice of that domain". But by missing the point that immersion is an essential part of the definition of interactional expertise, he threatens to draw the teeth of whole enterprise. This is at odds with the fundamentals of the SEE framework. 
Goddiksen is right in pointing out that to acquire interactional expertise there is a developmental phase, in which individuals might have little contact with high-level experts. In this phase, in which individuals could be said to be acquiring what he calls 'basic interactional expertise', their limited competence could be useful, enabling them to have a restricted understanding of the talk of full-blown experts and to make themselves intelligible to them (assuming the experts are willing to put in the necessary effort). The same could also be said of what Collins and Evans call primary source knowledge, which consists of knowledge that is learned through reading primary literature without any contact with the relevant community of experts, though this way of acquiring expertise is fraught with risk. This is because, as Collins and Evans have insisted from the outset, achieving interactional expertise means becoming a fully accomplished speaker of a domain language and this involves much more than learning a vocabulary or a set of standard skills. By virtue of being able to use the practice language, interactional experts are able to make a number of distinctions within a domain of practice, such as what papers are relevant, what papers are outdated, and so forth. Individuals with primary source knowledge or 'basic interactional expertise' would not be able to make these judgements with the same authority and are much more likely to get them wrong, sometimes with serious consequences (Weinel 2007).

Sociology of science has empirically demonstrated that socialisation into a research community - not technical proficiency - is crucial for a scientist to become a full-blown contributory expert to a field of knowledge (e.g. Pinch 1986 and Kennefick 2000). In fact, as Collins (2011: 296, note 35) has pointed out, in the field of gravitational wave physics it is more likely that an interactional expert like him would be able to identify what is a relevant problem (though perhaps not a relevant solution) to the field than a partly-socialised graduate physics student, who would only know the technical, textbook 'tricks of the trade' needed to solve the idealised problems. This is why learning science for application in a real research environment is akin to an expert-guided artisan apprenticeship process (Delamont \& Atkinson 2001). The above does not mean that technical connoisseurship is not intrinsic to (contributory) expertise it is a necessary but not sufficient step - as Goddiksen himself points out in the first section. Historical studies on modern physics practice and pedagogy also support the view that it is personal interaction and immersion within localised theoretical cultures that allows mathematical tools and techniques to be appropriated beyond 'standard' technical competence and to then be modified to contribute novel developments to the field (Kaiser 2005). The motivation for Collins and Evans's work can be traced to Collins's previous sociological explorations of tacit knowledge (Collins 1974, Collins 1992) and to the philosophical answer that was written as a result of these investigations (Collins 2010, Collins \& Kusch 1998). These perspectives on tacit knowledge are fundamental to understanding why and how Goddiksen is led astray by the rejection of the 'immersion criterion'. Collins (2010) made several important advances in respect to the work of Polanyi, with the most salient one for the matter at hand being the distinction between three types of tacit knowledge: relational (knowledge that could be made explicit but, for whatever idiosyncratic or practical reason, is not), somatic ('embodied' knowledge in the style of Polanyi or Merleau-Ponty) and collective (knowledge that is maintained by and acquired through immersion in a social group).

All these types of tacit knowledge enter into the acquisition and transmission of expertise, but are seldom differentiated by most authors, and this can lead to problems when discussing 'tacit knowledge' as such. Goddiksen is right in that one acquires expertise by learning the technical tricks of the trade in solving a physics problem by doing the problem, finding the critical step which is not obvious and solving it. That is, by making the relational tacit knowledge explicit; certainly a sine qua non of contributory expertise. Also right is that one does not need interaction with a high-level contributory expert to do it. More often than not the answer to a textbook scientific problem can be found by consulting one's peers who have solved the problem, going to a TA's session, or simply through dogged persistence. Enough has been written about the somatic, so suffice it to say that there is no reason in principle why a lone person could not be able to mimic mechanical proficiency if enough time could be had to exposures to proficient performances. 
Learning to 'solve exercises on a blackboard proficiently', like 'playing chess proficiently', is an example of what physicists call 'acquiring physical intuition', which in reality means increasing one's intellectually-oriented somatic tacit knowledge (Reyes-Galindo 2011). Although in practice most scientific education is carried out by experts directly aiding student in the acquisition of the relational and the somatic, in theory one could dispense with experts for philosophical argumentation. So again it is right to say that to become a contributory expert high level of the somatic tacit are necessary and can be acquired without full immersion. Up to this point, Goddiksen's argument seems to hold.

Nevertheless, Goddiksen's rejection of the role of enculturation directly entails the rejection of the role of the collective tacit: whether it be performance in a lab (Collins 2010), working at a purely theoretical level (Reyes-Galindo 2011), or applying knowledge in industry (Ribeiro 2013), sociologists have shown that (unsurprisingly by its very nature) it is impossible to gain collective tacit knowledge without immersion. Also well-known to sociology is that not being enculturated into a group is an almost insurmountable barrier to contribute meaningfully to it, or in Goddiksen's terms, to 'advancing' a scientific field. ${ }^{3}$ In fact, when technically competent outsiders that have not been socialised into a community try to contribute to it, the harder they try to contribute the more they find themselves on the slippery-slope of being violently segregated and labelled as outcasts or 'cranks' by the community they aim to contribute to (particularly in physics, see Reyes-Galindo 2011 and Collins 2014).

The same argument can also be made from a late-Wittgensteinian perspective on what it means for an answer to a problem to be relevant. There may be many 'proper' answers to a scientific problem (there often are), but it is the rules of the language game or, in other words, the rules of the scientific community and the institutionalised way in which they are interpreted (Bloor 1997) - because modern science is a collective enterprise and not an individualistic one - that will determine not only what is the right answer, but indeed what the right question is. So despite the positive aspects of Goddiksen's pressing of the definition of interactional expertise, the claim that "there seems to be no principled reason to think that basic interactional expertise cannot be gained through primary sources only" does not hold under either empirical or philosophical analysis.

\section{Concluding Remarks}

Goddiksen's attempt to clarify Collins and Evans' concepts has brought up interesting questions regarding our understanding of contributory expertise that resonate with current SEE research perspectives, particularly when he seeks to work out what types of contribution to a scientific domain could lead to authorship of scientific papers. On the other hand, when trying to develop an epistemological demarcation criterion for contributory expertise the effort is much less successful and slips into a somewhat out-dated philosophical discussion. Also incorrect is Goddiksen's attempt to redefine interactional expertise as a phenomenon that does not rely on shared frames of meaning. By missing the central role of collective tacit knowledge in understanding the nature of expertise Goddiksen's proposal miss-represents what was originally proposed and does not offer any significant conceptual or empirical benefit. In sum, while Goddiksen's questions can lead to new and welcome developments regarding expertise, this will only be successful if the role of immersion, enculturation and socialisation as the means of acquiring collective tacit knowledge remain central to any refinement of the concepts.

\section{Acknowledgements}

We would like to thank Prof. Harry Collins and Dr. Robert Evans for commenting on preliminary versions of this paper. Dr. Reyes-Galindo's work is funded by the British Academy's Post Doctoral Fellowship scheme and Dr. Duarte's by a postdoctoral research project at the University of Brasilia. 
References

Bloor, D. (1997). Wittgenstein, rules and institutions. London and New York: Routledge.

Collins, H. M. (1974). The TEA set: Tacit knowledge and scientific networks. Science studies, 165185.

Collins, H. M. (1992). Changing order: Replication and induction in scientific practice. University of Chicago Press.

Collins, H. M. (1998). The Meaning of Data: Open and Closed Evidential Cultures in the Search for Gravitational Waves 1. American Journal of Sociology, 104(2), 293-338.

Collins, H. M. (2010). Tacit and explicit knowledge. University of Chicago Press.

Collins, H. M. (2011). Language and practice. Social Studies of Science, 41(2), 271-300.

Collins, H. M., \& Evans, R. (2002). The Third Wave of Science Studies: Studies of Expertise and

Experience. Social Studies of Science, 32(2), 235-296.

Collins, H. M., \& Evans, R. (2007). Rethinking expertise. University of Chicago Press.

Collins, H. M. (2014). Rejecting knowledge claims inside and outside science. Social Studies of Science, 0306312714536011.

Collins, H. M., Evans, R., \& Gorman, M. (2007). Trading zones and interactional expertise. Studies in History and Philosophy of Science Part A, 38(4), 657-666.

Collins, H. M., \& Kusch, M. (1998). The shape of actions: What humans and machines can do. MIT press.

Collins, H. M., and Sanders, G. (2007), They Give You the Keys and Say "Drive It:" Managers, Referred Expertise, and Other Expertises, in Collins (ed) Case Studies of Expertise and Experience: special issue of Studies in History and Philosophy of Science, 38, 4, 621-641.

Collins, H.M., Evans, R., Ribeiro, R., \& Hall, M. (2006). Experiments with interactional expertise. Studies in History and Philosophy of Science Part A, 37(4), 656-674.

Dasgupta, D., \& Levine, A. (2013). Trading zones, peripheral science and contributory expertise: a framework for exploring science outside the Western contexts. Current Science, 104(12), 1616-1618.

Delamont, S., \& Atkinson, P. (2001). Doctoring uncertainty: Mastering craft knowledge. Social Studies of Science, 31(1), 87-107.

Duarte, T. (2013). Expertise and the Fractal Model: Communication and Collaboration between Climate-Change Scientists. Cardiff University School of Social Sciences. Unpublished PhD Thesis.

Duarte, T. (2014a). Scientific Expertise from the Viewpoint of the Studies of Expertise and Experience. Paper presented at the VIII International Workshop on the Studies of Expertise and Experience, Kitchener, 9-12, June 2014.

Duarte, T. (2014b). Who's entitled to scientific authorship? A study of authorship rules in Paleoclimatology (in preparation).

Galison, P. (1997). Image and logic: A material culture of microphysics. University of Chicago Press.

Goddiksen, M. (2014). Clarifying interactional and contributory expertise. Studies in History and Philosophy of Science Part A, 47, 111-117.).

Kaiser, D. (2005). Drawing theories apart: the dispersion of Feynman diagrams in postwar physics. University of Chicago Press: Chicago

Kennefick, D. (2000). Star Crushing: Theoretical Practice and the Theoreticians' Regress. Social Studies of Science, 30(1), 5-40.

Pinch, T. J. (1986). Confronting nature: the sociology of solar-neutrino detection. Dordrecht: Holland.

Reyes-Galindo, L. (2011). The sociology of theoretical physics. Cardiff University School of Social Sciences. Unpublished PhD Thesis. 
Ribeiro, R. (2007). The language barrier as an aid to communication. Social Studies of Science, $37(4), 561-584$.

Ribeiro, R. (2013) Tacit Knowledge Management. Phenomenology and the Cognitive Sciences, 12(2), 337-366.

Star, S. L., \& Griesemer, J. R. (1989). Institutional ecology, translations and boundary objects: Amateurs and professionals in Berkeley's Museum of Vertebrate Zoology, 1907-39. Social Studies of science, 19(3), 387-420.

Weinel, M. (2007). Primary source knowledge and technical decision-making: Mbeki and the AZT debate. Studies In History and Philosophy of Science Part A 38(4), 748-760.

${ }^{1}$ See Collins et al (2006) for a description of the Imitation Game and its relevance to our understanding of tacit knowledge.

2 See Collins \& Kusch (1998) for an extensive discussion of why a conversation is a type of polimorphic action from which the collective and intentional cannot be subtracted in order to judge proficiency. Within that same framework, Goddiksen's conceptualisation of Imitation Game dialogues is closer to that of a mimeomorphic action in which intention and frames of collective meaning can be ignored when judging proficiency.

${ }^{3}$ This does not mean, of course, that outsiders cannot contribute to a field at all. The pathway $\mathrm{t}$ becoming a contributory expert could indeed be initially set out using a type of trading zone; see for example Dasgupta \& Levine (2013). 